\title{
AN ESTIMATE FROM BELOW FOR THE MARKOV CONSTANT OF A CANTOR REPELLER
}

\author{
ALEXANDER VOLBERG \\ Department of Mathematics, Michigan State University \\ East Lansing, Michigan 48824, U.S.A.
}

1. Here we will be concerned with the following version of the classical Markov inequality:

$$
\left\|p^{\prime}\right\|_{J} \leq M(\operatorname{deg} p)^{r}\|p\|_{J}
$$

where \|\|$_{J}$ stands for the supremum norm on compact $J, J \subset \mathbb{C}$, and $p$ denotes complex polynomials.

So we deal with a particular case of the inequality

$$
\left\|D^{\alpha} p\right\|_{E} \leq M(\operatorname{deg} p)^{r|\alpha|}\|p\|_{E}
$$

$E$ is a compact in $\mathbb{R}^{n}, p$ is a polynomial of $n$ real variables.

When $J$ lies on a line in $\mathbb{C}(1)$ becomes exactly the one-dimensional version of (2).

Let us start with mentioning that the description of compacts $E$ (or $J$ ) satisfying (2) is not known.

Some partial results are known. W. Pawłucki and W. Pleśniak [1], [2] showed that (2) holds whenever $E$ is uniformly polynomially cuspidal (UPC). In particular, every subanalytic compact set $E$ with $E=\overline{\operatorname{int} E}$ being UPC satisfies (2).

$J$. Siciak gave an important sufficient condition for (2). Let us consider $E \subset$ $\mathbb{R}^{n} \subset \mathbb{C}^{n}$. For (2) to hold, it is sufficient that the function

$$
G_{E}(a)=\sup \left\{\frac{1}{\operatorname{deg} p} \log |p(z)|: p \text { is a polynomial on } \mathbb{C}^{n}, \operatorname{deg} p \geq 1,\|p\|_{E} \leq 1\right\}
$$

satisfies the following estimate

$$
G_{E}(z) \leq M d(z)^{m}, \quad d(z) \stackrel{\text { def }}{=} \operatorname{dist}(z, E) .
$$

1991 Mathematics Subject Classification: Primary 42A50.

The paper is in final form and no version of it will be published elsewhere. 
Moreover, the constants in (2) and (3) are connected by an inequality

$$
r \leq m^{-1} \text {. }
$$

Siciak constructed a Cantor type set on $\mathbb{R}$ which satisfies (3). This shows that the class of sets for which (2) holds is strictly larger than UPC.

Using Siciak's implication $(3) \Rightarrow(2)$ L. Białas and the author showed in [4] that standard Cantor sets on $\mathbb{R}$ have property (3), so (1). It is not clear how necessary Siciak's condition is for Markov's inequality.

Let us remind how the inequalities (2), (1) can be applied. In [1], [2] it was proved that Markov's inequality is equivalent to the existence on linear continuous right inverse to the restriction operator $C^{\infty}\left(\mathbb{R}^{n}\right) \ni f \rightarrow f \mid E$. Another way of expressing this is as follows. Let $\mathcal{P}_{\ell}$ denote all polynomials of degree $\ell$. If $\operatorname{dist}_{E}\left(f, \mathcal{P}_{\ell}\right)$ tends to zero faster than any $\ell^{-n}$ then $f$ is a restriction on $E$ of a function from $C^{\infty}\left(\mathbb{R}^{n}\right)$. This form of Bernstein's theorem is also proved by W. Pleśniak.

The goal of this paper is to get some estimates on $m$ and $r$ when $J$ is a Cantor repeller in $\mathbb{C}$.

2. Cantor repellers. Let $U, U_{1}, \ldots, U_{d}$ be topological discs with real analytic boundaries such that $\bar{U}_{i} \subset U, i=1, \ldots, d$. Consider a map $f: \bigcup_{i=1}^{d} U_{i} \rightarrow U$ which is univalent on $\bar{U}_{i}, i=1, \ldots, d$, and is a conformal isomorphism $f_{i}: U_{i} \rightarrow U$ on each $U_{i}$. By Cantor repeller we mean the set

$$
J=J(f)=\left\{x \in \mathbb{C}: f^{n} x \in U, n=0,1, \ldots\right\} .
$$

When $f=p=$ polynomial this is a Julia set of $p$.

We will also need the following notations. Let $G$ denote Green's function of $\Omega=\tilde{\mathbb{C}} \backslash J$ with pole at infinity, and let $\omega=\Delta G$ denote the harmonic measure of $J$. Let $\partial=\operatorname{dim}_{H} J$,

$$
\partial_{0}=\inf \left\{\operatorname{dim}_{H} J^{\prime}, J^{\prime} \text { is a Borel subset of } J, \omega\left(J^{\prime}\right)=1\right\} .
$$

The symbol $\operatorname{dim}_{H}$ denotes the Hausdorff dimension. There is a vast literature concerning the estimates of $\partial_{0}$. In particular, in [5] it is shown that always $\partial_{0} \leq 1$. And in [6] the estimate

$$
\partial_{0}<\partial
$$

is proved for the case $f_{i}(\bar{z})=\overline{f_{i}(z)}, U_{i} \cap \mathbb{R} \neq \phi$. Also (5) holds when $f=p=$ polynomial, see [7].

3. Main results. We are going to give some estimates of $m$ from above and (in the polynomial case) for $r$ from below.

TheOREM 3.1. Let $\left(U, U_{1}, \ldots, U_{d}, f\right)$ define a Cantor repeller $J=$ $\bigcap_{n \geq 0} f^{-n}(U)$. Let $m$ satisfy (3) with $E=J$. Then the following assertions are equivalent:

1) any such $m$ is strictly less than $\partial$; 
2) any such $m$ is strictly less than $\partial_{0}$

3) $\partial_{0}<\partial$.

Remark. The author believes that $\partial_{0}<\partial$ for any Cantor repeller. However, let us remind that (5) is still proved only for $f=$ polynomial [7] and for symmetric $f[8]$, or "linear" $f[11]$.

For polynomial case one can give the estimate not only for $m$ but also for $r$.

TheOREM 3.2. Let $J$ be a Julia set of a polynomial p. Then the Markov's inequality (1) holds only with

$$
r \geq \frac{1}{\partial_{0}}
$$

Remark. Let us note that $J$ is not assumed to be a Cantor repeller.

Next is considered a "standard Cantor set" situation. And we illustrate how one can easily see that $m<\partial_{0}$. Then

TheOrem 3.3. Let $J=J(f), f=\left(f_{1}, \ldots, f_{d}\right) ;\left|f_{i}^{\prime}(z)\right| \equiv l, z \in U_{i}, i=1, \ldots, d$.

$$
m<\partial_{0}
$$

4. Gibbs measures. It will be convenient to use the notion of special class of ergodic measures, namely the class of Gibbs measures in what follows. The reader may refer to [8] for the theory of these measures.

Let $J=J(f), f=\left(f_{1}, \ldots, f_{d}\right)$ be a Cantor repeller and let $f^{*}$ denote the pushing forward of the measures on $J$. What we mean is the following. Let $\nu$ be a measure on $J$. Then $f^{*} \nu$ is defined on $J \cap U_{i}$ as $\left(f^{*} \nu\right)(E)=\nu\left(f_{i} E\right)$. A measure $\nu$ will be called quasi-invariant if $\nu$ is mutually absolutely continuous with respect to $f^{*} \nu$. For any quasi-invariant measure $\nu$ on $J$ let us consider its Jacobian

$$
\mathcal{G}_{\nu}(x)=\frac{d f^{*} \nu}{d \nu}(x), \quad \text { for } \nu \text {-a.e. } x \in J
$$

which is the derivative of $f$ with respect to $\nu$. The function $\varphi_{\nu}(x) \stackrel{\text { def }}{=}-\log \mathcal{G}_{\nu}(x)$ will be called the potential of $\nu$. Generally speaking Gibbs measures are those having Hölder potentials. Let us state the definition and some properties of Gibbs measures.

1) By a Gibbs measure on $J(f)$ we mean an $f$-invariant $\mu$ with Hölder potential $\varphi_{\mu}=-\log \mathcal{G}_{\mu}$.

2) If $\nu$ is an $f$-quasi-invariant measure with Hölder potential, then there exists a unique $f$-invariant $\mu$ absolutely continuous with respect to $\nu$. Also $\mu$ is a Gibbs measure and $\log \frac{d \nu}{d \mu}$ is Hölder continuous. 
3) For any Hölder continuous $\varphi$ there exists a unique measure $\mu$ which provides the maximum for the functional

$$
L_{\varphi}(\nu)=h_{\nu}+\int_{J} \varphi d \nu, \nu \text { is } f \text {-invariant, probabilistic }
$$

(here $h_{\nu}$ denotes the entropy). This measure $\mu$ is Gibbs. Any other Hölder function $\psi$ defines the same $\mu$ if and only if

$$
\varphi-\psi=\gamma \circ f-\gamma+C,
$$

where $C$ is a constant and $\gamma$ is Hölder continuous.

Equation (9) is called the homologous equation. If we define $P(\varphi)=\max L_{\varphi}(\nu)$ (called pressure of $\varphi$ ) then the constant $C$ in $(9)$ is $P(\varphi)-P(\psi)$.

4) If $\mu$ is a Gibbs measure on $J$, then $X_{n}(x)=\left\{i: f^{n} x \in U_{i}\right\}$ form the sequence of exponentially independent random variables on $(J, \mu)$, in particular

$$
\left|\mathbb{E} X_{n} X_{n+k}-\mathbb{E} X_{n} \mathbb{E} X_{n+k}\right| \leq C q^{k}, \quad q \in(0,1) .
$$

The property 4) will be used soon. We need the following corollary of (1).

THEOREM 4.1. Let $\xi$ be a Hölder continuous function on $J$. Let $\mu$ be a Gibbs measure $\int \xi d \mu=0$ and let $\left\{Y_{n}\right\}$ denote the sequence of random variables on $(J, \mu)$ defined as follows

$$
Y_{n}=\xi\left(f^{n} x\right), \quad x \in J, n=0,1, \ldots
$$

Then $\left\{Y_{n}\right\}$ are exponentially independent. In particular the limit

$$
\sigma^{2}=\lim \frac{1}{n} \mathbb{E}\left(Y_{1}+\ldots+Y_{n}\right)^{2}
$$

exists and if $\sigma>0$ the law of iterated logarithm (LIL) holds for $\left\{Y_{n}\right\}$, namely

$$
\begin{aligned}
& \mu\left\{x: Y_{1}+\ldots+Y_{n}<-\sqrt{2 \sigma^{2} n \log \log n} \text { for infinitely many } n\right\}=1 \\
& \mu\left\{x: Y_{1}+\ldots+Y_{n}>+\sqrt{2 \sigma^{2} n \log \log n} \text { for infinitely many } n\right\}=1
\end{aligned}
$$

Theorem 4.1 can be found in [9].

At the same time a result of Ibragimov [10] describes the cases when $\sigma=0$.

THEOREM 4.2. Let $\sigma=0$. Then

$$
Y=u \circ f-u
$$

where $u \in L^{2}(J, d \mu)$.

5. Proof of Theorem 3.1. Let $G$ be Green's function of $\mathbb{C} \backslash J$ (that trivially coincides with Siciak's function defined before (3)). It is proved in [6] that the harmonic measure $\omega=\Delta G$ of $J$ is $f$ quasi-invariant and has Hölder potential $\varphi_{\omega}=-\log \mathcal{G}_{\omega}$. According to Section 4 there exists a Gibbs measure $\mu_{0}$ equivalent with $\omega$. 
Let $\varphi$ denote the Hölder function $\log \mathcal{G}_{\mu_{0}}, \psi \stackrel{\text { def }}{=} \log \left|f^{\prime}\right|$ which is certainly Hölder. Finally

$$
\xi \stackrel{\text { def }}{=} \varphi-m \psi, \quad Y_{k}=\xi \circ f^{k} .
$$

LEMMA 5.1. Let $U_{n}(x)$ denote the component of $f^{-n} U$ containing $x \in J$. Then

$$
\begin{gathered}
\operatorname{diam} U_{n}(x) \asymp\left|\left(f^{n}\right)^{\prime}(x)\right|^{-1} ; \quad d(z, J) \asymp \operatorname{diam} U_{n}(x), \quad z \in \partial U_{n}(x), \\
\mu_{0}\left(U_{n}(x)\right) \asymp \sup _{z \in U_{n}(x)} G(z) .
\end{gathered}
$$

This is proved in [6].

Lemma 5.2. $\left|\log \mu_{0}\left(U_{n}(x)\right)+\sum_{k=1}^{n} \varphi\left(f^{k} x\right)\right| \leq C$.

This follows immediately from the fact that $\varphi$ is the potential of $\mu_{0}$ and from the Hölder continuity of $\varphi$. On the other hand, one can derive Lemma 5.2 from the alternative description of Gibbs measures in [8].

Now we may rewrite (3) as

$$
\sum_{1}^{n} Y_{k}=\sum_{1}^{n} \varphi\left(f^{k} x\right)-m \sum_{1}^{n} \log \left|f^{\prime}\left(f^{k} x\right)\right| \geq C_{1} .
$$

We use Lemmas 5.1, 5.2 to obtain (13) from (3).

On the other hand $\int_{J}\left(\varphi-\partial_{0} \psi\right) d \mu_{0}=0$. This is just the form of writing Manning's formula for the dimension of the $f$-invariant measure $\mu_{0}$ :

$$
\partial_{0}=\operatorname{dim} \mu_{0}=\frac{h \mu_{0}}{\int_{J} \log \left|f^{\prime}\right| d \mu_{0}}=\frac{\int \varphi d \mu_{0}}{\int \log \left|f^{\prime}\right| d \mu_{0}} .
$$

Thus if $\partial_{0}$ were equal to $m$ we would have

$$
\mathbb{E} Y_{k}=\int \xi d \mu=\int(\varphi-m \psi) d \mu_{0}=\int\left(\varphi-\partial_{0} \psi\right) d \mu_{0}=0 .
$$

this would bring us under the conditions of Theorem 4.1, 4.2. But (13) contradicts LIL of Theorem 4.1. We conclude that $\sigma^{2}=\lim _{n \rightarrow \infty} \frac{1}{n} \mathbb{E}\left(Y_{1}+\ldots+Y_{n}\right)^{2}=0$ which means that

$$
\log \mathcal{G}_{\mu_{0}}-\partial_{0} \log \left|f^{\prime}\right|=\varphi-m \psi=u \circ f-u .
$$

In particular, using (9) we obtain

$$
P\left(-\partial_{0} \log \left|f^{\prime}\right|\right)=0 .
$$

But by Bowen's theorem the unique $\partial$ such that $P\left(-\partial \log \left|f^{\prime}\right|\right)=0$ is the Hausdorff dimension of $J$. Thus $\partial_{0}=\partial$. The implication 3$) \Rightarrow 2$ ) is proved.

This was the most difficult implication. Clearly $m \leq \partial_{0} \leq \partial((3)$ shows that $H_{m-\varepsilon}(E)=\infty$ as soon as $\left.\omega(E)>0 \Rightarrow \partial_{0}=\operatorname{dim} \omega \geq m-\varepsilon\right)$. So 2$\left.) \Rightarrow 1\right)$ is trivial.

The implication 1$) \Rightarrow 3$ ). This repeats the consideration in [6]. If $\partial_{0}=\partial$ then Manning's formula (14) gives

$$
h_{\mu_{0}}-\partial \int \log \left|f^{\prime}\right| d \mu_{0}=0
$$


So $\mu_{0}$ is the measure giving the maximum $P\left(-\partial \log \left|f^{\prime}\right|\right)=0$ to the functional $L_{\partial \log \left|f^{\prime}\right|}(\nu)=h_{\nu}-\partial \int \log \left|f^{\prime}\right| d \nu$. We see that $\mu_{0}$ is the Gibbs measure constructed by means of $-\partial \log \left|f^{\prime}\right|$. Now using (9) we come to the homologous equation (15) with Hölder $u$. Lemma 5.1 shows that $G(z) \leq M d(z)^{\partial}$. This contradicts 1$)$ and $1) \Rightarrow 3$ ) is proved together with Theorem 3.1.

Let $m_{0}=\sup \{m:(3)$ is satisfied with a certain $M=M(m)<\infty\}$. Then clearly

$$
m_{0}=\lim _{z \rightarrow J} \frac{\log G(z)}{\log d(z)} .
$$

QUeSTION 1. Is always $m_{0}<\partial_{0}$ ?

6. A simple estimate for $m_{0}$ from above. Let us consider the following expression in which supremum is taken over all ergodic measures on $J$ :

$$
r_{1} \stackrel{\text { def }}{=} \sup _{\mu} \frac{\int \log \left|f^{\prime}\right| d \mu}{\int \log \mathcal{G}_{\omega} d \mu} .
$$

Let $\nu$ provide "almost" supremum to this expression. Then by the ergodic theorem we get that

$$
\omega\left(U_{n}(x)\right) \geq C\left(\operatorname{diam} U_{n}(x)\right)^{\frac{1}{r_{1}}+\varepsilon}, \quad \text { for } \nu \text {-a.e. } x .
$$

Using Lemma 5.1 we derive immediately the estimate

$$
\frac{1}{m_{0}} \geq r_{1} \text {. }
$$

7. Polynomial case. For $f=p=$ polynomial we can write $r_{1}$ as follows:

$$
r_{1}=\frac{1}{\log d} \sup _{\mu} \int \log \left|p^{\prime}\right| d \mu \stackrel{\text { def }}{=} \frac{\sup \chi_{\mu}}{\log d} .
$$

Let us introduce $r_{0}=\inf \{r:(1)$ is satisfied $\}$.

LEMMA 7.1. $\frac{1}{m_{0}} \geq r_{0} \geq r_{1}$.

Pr o of. The first inequality is a trivial consequence of (4). To prove the second let us introduce

$$
\chi_{p}(x)=\varlimsup_{n \rightarrow \infty} \frac{1}{n} \log \left|\left(p^{n}\right)^{\prime}(x)\right| \quad \text { and } \quad r_{s}=\frac{\sup _{x \in J} \chi_{p}(x)}{\log d} .
$$

The functions $f_{n}=\frac{1}{n} \log \left|\left(p^{n}\right)^{\prime}(x)\right|$ are bounded from above (but maybe not from below if $p^{\prime}$ vanishes on $J$ ) and so for them $\overline{\lim } \int f_{n} \leq \int \varlimsup \lim f_{n}$. So

$$
\begin{aligned}
\int \log \left|p^{\prime}\right| d \mu & =\frac{1}{n} \int \log \left|\left(p^{n}\right)^{\prime}\right| d \mu=\varlimsup \\
& \leq \int \varlimsup \frac{1}{n} \log \left|\left(p^{n}\right)^{\prime}\right| d \mu \\
& \leq \frac{1}{n} \log \left|\left(p^{n}\right)^{\prime}\right| d \mu \leq \sup _{x \in J} \chi_{p}(x) .
\end{aligned}
$$


Thus

$$
r_{1} \leq r_{s}
$$

On the other hand (1) shows that

$$
\left|\left(p^{n}\right)^{\prime}(x)\right| \leq M\left(d^{n}\right)^{r_{0}+\varepsilon}
$$

and so for each $x \in J$

$$
\chi_{p}(x) \leq\left(r_{0}+\varepsilon\right) \log d
$$

which means that $r_{s} \leq r_{0}$. Combining this with (16) we complete the proof of Lemma 7.1.

Let us introduce

$$
\chi_{p}^{\text {orb }}=\sup \left\{\chi_{p}(x): x \text { is a periodic point of } p\right\} .
$$

LEMmA 7.2. $\sup _{\mu} \chi_{\mu} \leq \chi_{p}^{\text {orb }} \leq \sup _{x \in J} \chi_{p}(x)$, where the first supremum is taken over all ergodic measures.

We will not use this refinement of (16) and we cite this fact about Lyapunov exponents only for the sake of completeness.

8. Proof of Theorem 3.2. Just choose $\mu=\mu_{0}$ to estimate $r_{1}$. Then

$$
r \geq r_{1} \geq \frac{\int \log \left|p^{\prime}\right| d \mu_{0}}{\log d}=\frac{1}{\partial_{0}} .
$$

The last equality is (14)

Now [7] and Theorem 3.1 show that either $\frac{1}{m}>r$ or $r>\frac{1}{\partial_{0}}$.

9. Proof of Theorem 3.3. Here to prove (7) we will need the following:

LemMa 9.1. Let $\varphi$ be a Hölder function on $J$ such that $\varphi$ is not homologous to a constant. Let $\alpha_{t}$ be the Gibbs measure for $t \varphi ; \alpha=\alpha_{1}$. Then

$$
\left(\int \varphi d \alpha_{t}-\int \varphi d \alpha\right)(1-t)>0 \text {. }
$$

Proof. We use 3) of Section 4 to write

$$
\begin{gathered}
h_{\alpha}-\int \varphi d \alpha>h_{\alpha_{t}}-\int \varphi d \alpha_{t} \\
h_{\alpha_{t}}-t \int \varphi d \alpha_{t}>h_{\alpha}-\int \varphi d \alpha .
\end{gathered}
$$

Subtracting these two lines we get the result.

Now let $\varphi=\log \mathcal{G}_{\mu_{0}}$ and $\alpha=\mu_{0}$. This $\varphi$ is not homologous to a constant [6]. Now Lemma 9.1 gives us an ergodic measure $\alpha_{t}$ such that

Thus

$$
\int \log \mathcal{G}_{\mu_{0}} d \alpha_{t}<\int \log \mathcal{G}_{\mu_{0}} d \mu_{0}=h_{\mu_{0}} .
$$

$$
\frac{1}{m} \geq r_{1} \geq \frac{\log l}{\int \log \mathcal{G}_{\mu_{0}} d \alpha_{t}}>\frac{\log l}{\int \log \mathcal{G}_{\mu_{0}} d \mu_{0}}=\frac{\int \log \left|f^{\prime}\right| d \mu_{0}}{h_{\mu_{0}}}=\frac{1}{\partial_{0}}
$$


QUESTION 2. In the polynomial case, is it true that $r>\frac{1}{\partial_{0}}$ for any $r$ satisfying Markov's inequality (1) unless $p=z^{k}$ ?

QUESTION 3. In the polynomial case, is it true that $r_{0}>\frac{1}{\partial_{0}}$ or even $r_{1}>\frac{1}{\partial_{0}}$ unless $p=z^{k}$ ?

Remark. The inequality $r_{1}>\frac{1}{\partial_{0}}$ means that harmonic measure on $J(p)$ does not provide the maximum for the functional $\nu \rightarrow \int \log \left|p^{\prime}\right| d \nu$ among ergodic measures.

\section{References}

[1] W. Pawłucki and W. Pleśniak, Extension of $C^{\infty}$ functions from sets with polynomial cusps, Studia Math. 88 (1988), 279-287.

[2] -, -, Prolongement de fonctions $C^{\infty}$, C.R. Acad. Sci. Paris Ser. I 304 (1987), 167-168.

[3] J. Siciak, On some extremal functions and their applications in the theory of analytic functions of several complex variables, Trans. Amer. Math. Soc. 105 (1962), 322-357.

[4] L. Białas and A. Volberg, Markov's property of the Cantor ternary set, Studia Math. 104 (1993), 259-268.

[5] P. Jones and Th. Wolff, Hausdorff dimension of harmonic measure in the plane I, Acta Math. 161 (1988), 133-144.

[6] A. Volberg, On the dimension of harmonic measure of Cantor repellers, Michigan Math. J. 40 (1993), 239-258.

[7] A. Zdunik, Parabolic orbifolds and the dimension of the maximal measure for rational maps, Invent. Math. 99 (1990), 627-649.

[8] R. Bowen, Equilibrium states and the ergodic theory of Anosov diffeomorphisms, Lect. Notes in Math. 470 (1975).

[9] W. Phillipp and W. Stout, Almost sure invariant principles for partial sums of weakly dependent random variables, Mem. Amer. Math. Soc. 161 (1975).

[10] I. A. Ibragimov and Yu. V. Linnik, Independent and stationary sequences of random variables, Wolters-Noordhoff, Groningen, 1971

[11] A. Volberg, On the harmonic measure of self-similar sets on the plane, in: Harmonic Analysis and Discrete Potential Theory, M. Picardello (ed.), Plenum Press, 1991, 267-281. 